\title{
PESO DO BAÇO COMO FATOR PROGNÓSTICO DO TRATAMENTO CIRÚRGICO DE PACIENTES PORTADORES DE ESQUISTOSSOMOSE MANSÔNICA
}

\author{
SPLEEN WEIGHT AS RISK FACTOR IN THE SURGICAL TREATMENT \\ OF PATIENTS WITH SCHISTOSOMA MANSONI
}

\author{
Álvaro Antônio Bandeira Ferraz TCBC-PE ${ }^{1}$ \\ Edmundo Pessoa de Almeida Lopes ${ }^{2}$ \\ José Guido Corrêa de Araújo Júnior ${ }^{3}$ \\ Bruno de Andrade Lima ${ }^{4}$ \\ Fabiano Cantarelli ${ }^{4}$ \\ Edmundo Machado Ferraz TCBC-PE ${ }^{5}$
}

\begin{abstract}
RESUMO: Objetivo: Analisar pré-operatoriamente o peso do baço como fator prognóstico do tratamento cirúrgico de pacientes portadores de esquistossomose mansônica. Métodos: Foram analisados 114 pacientes, portadores de esquistossomose mansônica com antecedentes de hemorragia digestiva, submetidos a tratamento cirúrgico. Os pacientes foram divididos em três grupos: Grupo 1 - baço menor que 500 gramas (17); Grupo 2 - baço entre 500 e 1.000 gramas (58); Grupo 3 - baço acima de 1.000 gramas (39). Resultados: No Grupo 1 a recidiva hemorrágica foi de $17,6 \%$, trombose da veia porta de $5,9 \%$ e não houve mortalidade. A incidência de hiperesplenismo pré-operatório foi de $29,4 \%$ e o calibre da veia porta foi de $1,1 \mathrm{~cm}$. No Grupo 2 a recidiva hemorrágica foi de $15,5 \%$, trombose da veia porta de $10,3 \%$ e a mortalidade de quatro pacientes $(6,9 \%)$ (dois pacientes no período pós-operatório e dois no seguimento tardio, hepatocarcinoma e hemorragia digestiva). A incidência de hiperesplenismo foi de $53,4 \%$ e o calibre médio da veia porta foi de $1,4 \mathrm{~cm}$. No Grupo 3 a recidiva hemorrágica foi de $12,8 \%$, trombose da veia porta de $5,1 \%$ e uma mortalidade tardia de dois pacientes (linfoma e infarto agudo do miocárdio). A incidência de hiperesplenismo foi de 76,9\% e o calibre da veia porta foi de $1,5 \mathrm{~cm}$. Conclusões: $\mathrm{O}$ peso do baço apresenta relação com o hiperesplenismo pré-operatório, calibre da veia porta e permanência hospitalar pós-operatória. Não encontramos relação com a incidência de varizes de fundo gástrico, recidiva de sangramento digestivo, trombose da veia porta, grau de fibrose periportal e dados bioquímicos.
\end{abstract}

Descritores: Esquistossomose hepatoesplênica; Esplenectomia; Baço; Fator de risco; Trombose da veia porta.

\section{INTRODUÇÃO}

O tratamento cirúrgico da hipertensão porta esquistossomótica através da esplenectomia com ligadura da veia gástrica esquerda, seguida de esclerose endoscópica pós- operatória, determina uma recidiva hemorrágica de $14,4 \%$ e uma mortalidade tardia de $5,4 \% 1$.

$\mathrm{Na}$ busca de identificar pré-operatoriamente fatores que possam comprometer os resultados cirúrgicos e talvez determinar condutas diferentes nestes pacientes, fo-

1. Professor Adjunto do Departamento de Cirurgia da Universidade Federal de Pernambuco (UFPE). Mestre e Doutor em Medicina pela UFPE. Especialização em Infecção em Cirurgia no Medical College of Wisconsin - EUA. Pós-doutorado em Transplante de Fígado na Universidade de Miami - EUA

2. Professor do Departamento de Medicina Clínica da UFPE

3. Médico Residente de 4o ano do Serviço de Cirurgia Geral do HC da UFPE

4. Aluno de Graduação do Curso de Medicina da UFPE

5. Professor Titular de Cirurgia Abdominal e Bases da Técnica Cirúrgica da UFPE Chefe do Serviço de Cirurgia Geral do Hospital das Clínicas da UFPE. Doutor e Livre-docente pela UFPE. Fellow do American College of Surgeons (FACS) e do Surgical Infection Society.

Recebido em 24/05/2001

Aceito para publicação em 27/11/2001

Trabalho realizado no Serviço de Cirurgia Geral do Hospital das Clínicas da Universidade Federal de Pernambuco. 
ram analisados os dados hematológicos, bioquímicos e ultra-sonográficos de 111 pacientes, sem que fatores de risco fossem identificados ${ }^{1,2}$.

Na fase aguda o baço é sede de fenômenos hiperplási$\cos _{\text {acentuados }}{ }^{3,4}$ que são acrescidos de alterações congestivas à medida que a hipertensão portal é estabelecida ${ }^{5}$.

$\mathrm{O}$ crescimento do baço é determinado na esquistossomose mansônica por duas variáveis:

Primeiro, a ação constante da pressão e estase sangüínea sobre a estrutura esponjosa do baço ${ }^{6,7} \mathrm{e}$, adicionalmente, um processo de hiperatividade imunológica ${ }^{8}$.

Além da imunodeficiência relativa ${ }^{6,8,9}$ na forma hepatoesplênica da esquistossomose, convivemos com esplenomegalias consideráveis, que poderiam influenciar os resultados cirúrgicos destes pacientes, visto que o tamanho do baço continua a ser considerado como fator prognóstico da morbidade da doença após instituído o tratamento medicamentoso específico ${ }^{10,11}$.

Os pacientes esquistossomóticos apresentam deficiências imunológicas importantes que, somadas a um estado nutricional comprometido e à magnitude do procedimento cirúrgico, o tornam um paciente de alto risco para complicações pós-operatórias ${ }^{7,9}$. Este dado tem representatividade clínica com o trabalho publicado por Ferraz et al. ${ }^{9}$ que comprovaram uma maior incidência de complicações infecciosas em pacientes portadores de esquistossomose hepatoesplênica.

$\mathrm{O}$ volume do baço não apresenta correlação com a severidade da hemorragia digestiva, mas apresenta correlação com o hiperesplenismo ${ }^{12}$.

A esplenectomia tem contribuído para reduzir de forma indiscutível a recidiva hemorrágica, principalmente quando associada a outros procedimentos cirúrgicos (ligadura de varizes esofagianas, ligadura da veia gástrica esquerda, ligadura das varizes de fundo gástrico) e endoscópicos $^{1}$. No entanto, a incidência de $14,4 \%$ de recidiva hemorrágica e a mortalidade de 5,6\% neste procedimento são consideradas pouco satisfatórios ${ }^{1,2}$.

$\mathrm{Na}$ tentativa de identificar fatores de risco que possam comprometer os resultados cirúrgicos é que analisamos o peso do baço como fator prognóstico no acompanhamento pós-operatório tardio.

\section{MÉTODOS}

No período de janeiro de 1992 a julho de 2000 foram acompanhados no Serviço de Cirurgia Geral do Hospital das Clínicas da Universidade Federal de Pernambuco 114 pacientes submetidos à esplenectomia com ligadura da veia gástrica esquerda (LVGE), desvascularização da grande curvatura do estômago e esclerose endoscópica pós-operatória, para o tratamento da hipertensão portal esquistossomótica com antecedentes de hemorragia digestiva.

Quando da presença de varizes de fundo gástrico (41/114) foi associada ao procedimento cirúrgico a abertura do estômago e sutura das varizes de fundo gástrico.

A esplenectomia segue uma padronização que inclui a abertura da retrocavidade do omento maior, abor- dagem e ligadura da artéria esplênica, liberação do pólo inferior do baço, ligadura dos vasos gastroesplênicos, liberação posterior do baço e abordagem do pedículo esplênico com ligadura e secção da veia esplênica e nova ligadura e secção da artéria esplênica.

Durante o procedimento cirúrgico foi realizada uma biopsia hepática em cunha de todos os pacientes, a qual foi compatível com esquistossomose mansônica pura. $\mathrm{O}$ baço foi pesado no momento da realização dos cortes para análise histopatológica. De acordo com o peso, dividimos a casuística em três grupos distintos: Grupo 1 - baço com peso inferior a 500 gramas; Grupo 2 - baço com peso entre 500 e 1.000 gramas e Grupo 3 - baço pesando mais de 1.000 gramas.

Todos os pacientes foram solicitados a retornar ao ambulatório de hipertensão portal e fígado do Hospital das Clínicas para a realização de exames hematológicos e bioquímicos seguidos de endoscopia e ultra-sonografia e ultra-sonografia doppler do fígado, para avaliação do fluxo portal.

O seguimento médio foi de 34,2 meses nos pacientes do Grupo 1, 30,1 meses nos pacientes do Grupo 2 e 28,1 meses nos pacientes do Grupo 3 .

Os 114 pacientes apresentavam epidemiologia positiva para a esquistossomose mansônica, caracterizada por banho de rio em área endêmica, assim como antecedentes de hemorragia digestiva exteriorizada por hematêmese $(102 / 114)$ ou por melena (12/114).

Os critérios de inclusão foram:

- idade superior a 16 anos;

- antecedente de hemorragia digestiva;

- varizes esofagianas ao exame endoscópico;

- hematócrito acima de $22 \mathrm{ml} / \mathrm{dl}$;

- tempo de protrombina e atividade enzimática não inferior a $50 \%$;

- exames de sorologia viral para hepatite negativos;

- confirmação da biopsia hepática de patologia esquistossomótica pura.

Os critérios de exclusão foram:

- hepatopatia mista;

- passado de alcolismo;

- trombose da veia porta.

A idade dos pacientes variou entre 22 e 75 anos, com uma média de 45,2 anos.

Os pacientes foram operados de maneira eletiva, fora do quadro agudo de hemorragia digestiva.

A classificação do grau de fibrose esquistossomótica obedeceu aos critérios descritos por Coelho ${ }^{13}$. De acordo com a sua intensidade o grau de fibrose foi classificado em:

- Grau 1 - os espaços portas apresentam-se com maior riqueza de células conjuntivas jovens, discreta produção de colágeno e presença variável de infiltrado inflamatório. A lâmina periportal e o retículo são normais.

- Grau 2 - há expansão do tecido conjuntivo com emissão de septos colágenos radiais, dando ao mesmo um aspecto estrelado. 
- Grau 3 - os septos conjuntivos formam pontes com outros espaços portas ou com a veia, havendo neoformação angiomatóide bem evidente.

Os critérios diagnósticos do hiperesplenismo secundário à hipertensão portal esquistossomótica foram: 1) anemia (hemoglobina menor que $13 \mathrm{~g} / \mathrm{dl}$ nos homens e menor que $12 \mathrm{~g} / \mathrm{dl}$ nas mulheres), leucopenia (menos de $1.500 \mathrm{~mm}^{3}$ leucócitos), trombocitopenia (menos de $150.000 \mathrm{~mm}^{3}$ plaquetas) ou a combinação destes; 2) esplenomegalia; 3) melhora ou regressão após a esplenectomia ${ }^{14,15}$.

$\mathrm{Na}$ análise estatística dos dados foi utilizado um teste de "t" para diferença de médias e Qui-quadrado, com nível de significância de $95 \%$.

\section{RESULTADOS}

Os grupos 1, 2 e 3 foram constituídos por 17, 58 e 39 pacientes, respectivamente.

O seguimento médio pós-operatório foi de 34,2 meses para os pacientes do Grupo 1, 30,1 meses para os pacientes do Grupo 2 e 28,1 meses nos pacientes do Grupo 3. Não houve diferença estatística entre os grupos.

A permanência hospitalar foi de 4,2, 7,6 e 7 dias, para os pacientes dos Grupos 1, 2 e 3, respectivamente. A permanência hospitalar dos pacientes do Grupo 1 foi estatisticamente menor quando comparada com a permanência dos Grupos 2 e 3.
A ocorrência de varizes de fundo gástrico não apresentou relação com o peso do baço. A ocorrência de varizes de fundo gástrico nos pacientes do Grupo 1 foi de $41,2 \%$ (7/17) e, nos pacientes do Grupo 2 e 3 foi de $34,5 \%$ (20/58) e $35,9 \%$ (14/39), respectivamente.

Com relação ao hiperesplenismo secundário à hipertensão portal esquistossomótica a prevalência no préoperatório foi estatisticamente maior nos pacientes que apresentaram baço com peso superior a 1.000 gramas (76,9\%), quando comparados com os pacientes do Grupo $2(53,4 \%)$ e com os pacientes do Grupo $1(29,4 \%)$.

Observou-se correlação entre o calibre da veia porta e o aumento do baço. Nos pacientes com baço pesando menos de 500 gramas o calibre médio da veia porta foi de $1,1 \mathrm{~cm}$. Nos pacientes com baço pesando entre $500-1.000$ gramas o calibre médio foi de $1,4 \mathrm{~cm}$ e nos pacientes em que o baço pesava mais de 1.000 gramas o calibre médio foi de $1,5 \mathrm{~cm}$.

A recidiva de hemorragia digestiva e a incidência de trombose da veia porta pós-operatória, no seguimento tardio, não apresentaram diferença em relação ao peso do baço. Assim como não encontramos relação entre o grau de fibrose periportal e o peso do baço (Tabela 1).

Não houve mortalidade no Grupo 1 entre os 17 pacientes operados. Nos pacientes do Grupo 2 houve quatro óbitos, tendo sido dois no pós-operatório imediato e dois no pós-operatório tardio. No pós-operatório imediato os dois óbitos foram em conseqüência de sepse e de coagulação intravascular disseminada. Os pacientes com morta-

Tabela 1

Relação entre o peso do baço e os resultados cirúrgicos de pacientes portadores de esquistossomose hepatoesplênica

\begin{tabular}{|c|c|c|c|}
\hline & & Peso do Baço & \\
\hline & $\begin{array}{c}\text { Grupo } 1 \\
<500 \text { gramas } \\
\left(N^{o}=17 \text { pac. }\right)\end{array}$ & $\begin{array}{c}\text { Grupo } 2 \\
500-1.000 \text { gramas } \\
\left(N^{\circ}=58 \text { pac. }\right)\end{array}$ & $\begin{array}{c}\text { Grupo } 3 \\
>1.000 \text { gramas } \\
(N N=39 \text { pac. })\end{array}$ \\
\hline Seguimento médio & 34,2 meses & 30,1 meses & 28,1 meses \\
\hline Permanência hospitalar & 4,2 dias & 7,6 dias & 7,0 dias* \\
\hline Varizes fundo gástrico pré-operatório & $07(41,2 \%)$ & $20 \quad(34,5 \%)$ & $14(35,9 \%)$ \\
\hline Hiperesplenismo pré-operatório & $05(29,4 \%)$ & $31 \quad(53,4 \%)$ & $30(76,9 \%)^{*}$ \\
\hline Calibre da veia porta & & & \\
\hline Pré-operatório & $1,1 \mathrm{~cm}$ & $1,4 \mathrm{~cm}$ & $1,5 \mathrm{~cm}^{*}$ \\
\hline Pós-operatório & $0,8 \mathrm{~cm}$ & $0,9 \mathrm{~cm}$ & $1,0 \mathrm{~cm}$ \\
\hline Recidiva sangramento & $03(17,6 \%)$ & $09 \quad(15,5 \%)$ & $05 \quad(12,8 \%)$ \\
\hline Trombose da veia porta & $01 \quad(5,9 \%)$ & $06 \quad(10,3 \%)$ & $02 \quad(5,1 \%)$ \\
\hline Fibrose periportal & & & \\
\hline Grau I & $02(11,8 \%)$ & $16(27,6 \%)$ & $08 \quad(20,5 \%)$ \\
\hline Grau II & $07(41,2 \%)$ & $21 \quad(36,2 \%)$ & $16(41,0 \%)$ \\
\hline Grau III & $08(47,0 \%)$ & $21 \quad(36,2 \%)$ & $15 \quad(38,5 \%)$ \\
\hline Mortalidade total & 0 & $(6,9 \%)$ & $(5,3 \%)$ \\
\hline Operatória & & $(3,4 \%)$ & 0 \\
\hline Tardia & & $02 \quad(3,4 \%)$ & $(5,3 \%)$ \\
\hline
\end{tabular}

$* p<0,05$ 
lidade tardia apresentaram diagnóstico de hemorragia digestiva e hepatocarcinoma. Dois pacientes do Grupo 3 foram a óbito no seguimento tardio com diagnóstico de linfoma não-Hodgkin e de infarto agudo do miocárdio.

$\mathrm{Na}$ análise bioquímica e hematológica dos pacientes no período pré-operatório ficou evidenciado, de maneira estatisticamente significativa, que os pacientes do Grupo 3 (peso do baço acima de 1.000 gramas), apresentavam valores de hematócrito, número total de leucócitos e linfócitos, plaquetas e tempo de protrombina, estatisticamente menores que os outros grupos estudados (Tabela 2). No entanto, o procedimento cirúrgico foi capaz de reverter estes dados de tal modo que no seguimento tardio não mais identificamos diferenças hematológicas ou bioquímicas nos grupos analisados (Tabela 3).

\section{DISCUSSÃO}

A grande polêmica relacionada ao tratamento cirúrgico da hipertensão porta esquistossomótica reside no fato de que há maneiras diferentes de se abordar o problema. Na tentativa de se reduzir ao máximo a recidiva hemorrágica realizam-se procedimentos que podem comprometer a função hepática e deteriorar a longo prazo o estado geral do paciente. Por outro lado, podem-se realizar procedimentos que têm pouca influência na função hepática, mas que cursam com uma recidiva hemorrágica mais elevada ${ }^{16}$.

Segundo Abrantes ${ }^{17}$, a operação ideal para tratar a hipertensão portal de origem esquistossomótica com an- tecedentes de hemorragia digestiva, deveria evitar a recidiva hemorrágica, não provocar encefalopatia, não agravar a função hepática e curar o hiperesplenismo.

Seguimos uma doutrina implantada e difundida pelo Prof. Salomão Kelner, desde os anos $1960^{18}$. Esta doutrina se baseava em um tratamento cirúrgico que atendesse à redução da pressão no compartimento portal, associado ao mínimo de alterações fisiológicas e que fosse capaz de reverter as alterações decorrentes do hiperesplenismo. Estamos seguindo e defendendo estes preceitos, associando apenas as novas tecnologias que tornaram acessíveis e os novos conhecimentos fisiopatológicos adquiridos. Deste modo, realizamos desde 1992 a esplenectomia com ligadura da veia gástrica esquerda associada à desvascularização da grande curvatura do estômago e posterior eslcerose endoscópica das varizes esofágicas ${ }^{1,2}$.

A esplenectomia na esquistossomose hepatoesplênica com antecedentes de hemorragia digestiva determina uma correção nos valores das alterações hematológicas (anemia, número de leucócitos, linfócitos e plaquetas), uma redução média da pressão portal na ordem de $28 \%$ e correção nos valores do hormônio do crescimento ${ }^{6,19,20}$.

$\mathrm{Na}$ tentativa de se identificar de maneira pré-operatória os pacientes que pouco se beneficiam do tratamento cirúrgico através da esplenectomia com ligadura da veia gástrica esquerda associada à desvascularização da grande curvatura do estômago, foram analisados dados hematológicos, bioquímicos, ultra-sonográficos e endoscópicos, sem que fosse identificado nenhum fator de risco $^{1,2}$. Em um total de 111 pacientes seguidos, notamos

Tabela 2

Relação entre o peso do baço e os dados hematológicos e bioquímicos de pacientes portadores de esquistossomose hepatoesplênica no período pré-operatório

\begin{tabular}{l|c|c|c}
\hline \multirow{2}{*}{} & \multicolumn{3}{c}{ Peso do Baço } \\
\cline { 2 - 4 } & $\begin{array}{c}\text { Grupo } 1 \\
500 \text { gramas } \\
\left(N^{\circ}=17 \text { pac. }\right)\end{array}$ & $\begin{array}{c}\text { Grupo } 2 \\
500-1.000 \text { gramas } \\
\left(N^{\circ}=58 \text { pac. }\right)\end{array}$ & $\begin{array}{c}\text { Grupo } 3 \\
1.000 \text { gramas } \\
\left(N^{\circ}=39 \text { pac. }\right)\end{array}$ \\
\hline Hematócrito & 33,1 & 32,1 & $29,3 *$ \\
Hemoglobina & 10,8 & 10,2 & 10,0 \\
Leucócitos & $5.437,5$ & $4.508,4$ & $3.403,9 *$ \\
Linfócitos & $1.263,1$ & 780,8 & $688,2 *$ \\
Plaquetas & $156.375,0$ & $121.992,8$ & $83.326,2^{*}$ \\
Tempo de protrombina1 & 3,4 & 14,4 & $16,2 *$ \\
Glicose & 95,4 & 100,0 & 94,7 \\
Uréia & 27,1 & 33,9 & 32,2 \\
Creatinina & 0,7 & 0,8 & 0,8 \\
Albumina & 3,5 & 3,7 & 3,7 \\
TGO & 39,4 & 38,4 & 36,3 \\
TGP & 31,0 & 34,8 & 35,6 \\
Bilirrubinas totais & 1,1 & 0,8 & 1,2
\end{tabular}

$* p<0,05$. 
Tabela 3

Relação entre o peso do baço e os dados hematológicos e bioquímicos de pacientes portadores de esquistossomose hepatoesplênica no período pós-operatório

\begin{tabular}{|c|c|c|c|}
\hline & \multicolumn{3}{|c|}{ Peso do Baço } \\
\hline & $\begin{array}{c}\text { Grupo } 1 \\
<500 \text { gramas } \\
\left(N^{o}=17 \text { pac. }\right)\end{array}$ & $\begin{array}{c}\text { Grupo } 2 \\
500-1.000 \text { gramas } \\
\left(N^{\circ}=58 \text { pac. }\right)\end{array}$ & $\begin{array}{c}\text { Grupo } 3 \\
>1.000 \text { gramas } \\
\left(N^{o}=39 \text { pac. }\right)\end{array}$ \\
\hline Hematócrito & 36,6 & 39,3 & 39,7 \\
\hline Hemoglobina & 12,0 & 13,1 & 13,1 \\
\hline Leucócitos & $6.788,9$ & $6.243,9$ & $8.421,1$ \\
\hline Linfócitos & $1.916,7$ & $1.952,0$ & $2.424,7$ \\
\hline Plaquetas & $277.111,1$ & $267.454,4$ & $347.371,6$ \\
\hline Tempo de protrombina & 12,4 & 12,9 & 12,6 \\
\hline Glicose & 97,8 & 96,6 & 105,1 \\
\hline Uréia & 25,1 & 36,2 & 25,2 \\
\hline Creatinina & 0,7 & 0,7 & 0,7 \\
\hline Albumina & 3,5 & 3,9 & 4,1 \\
\hline TGO & 51,0 & 52,4 & 51,3 \\
\hline TGP & 41,6 & 40,2 & 33,1 \\
\hline Bilirrubinas totais & 1,0 & 0,9 & 1,0 \\
\hline
\end{tabular}

que 16 pacientes apresentaram recidivas hemorrágicas. Destes 16 pacientes três apresentavam trombose da veia porta, três desenvolveram varizes de fundo gástrico no seguimento tardio, um paciente evoluiu com um hepatocarcinoma e posterior sangramento e um paciente apresentou hematêmese no pós-operatório imediato, indo a óbito.

$\mathrm{O}$ peso do baço pode influenciar os resultados de nossa proposta cirúrgica do tratamento da hipertensão portal esquistossomótica. A retirada de uma grande "massa" abdominal, que determinava relevante seqüestro de sangue e conseqüiente aumento da pressão portal, pelo hiperfluxo, poderia ser um fator de risco ao desenvolvimento de trombose da veia porta. Assim como baços de peso inferior a 500 gramas pouco se beneficiariam de sua retirada, visto que pouco reduziria a pressão portal. Pacientes que apresentam baços com peso acima de 1.000 gramas poderiam apresentar correlação com o grau de hipertensão portal ou com a ocorrência de varizes de fundo gástrico.

No entanto, com base em nossos resultados evidenciamos que:

1. A permanência hospitalar foi estatisticamente menor nos pacientes em que o baço pesava menos de 500 gramas. Isto reforça a tese de que estes pacientes apresentam menos alterações hematológicas e bioquímicas e deste modo o período pós-operatório foi mais tranqüilo e com menos complicações.

2. As varizes de fundo gástrico não apresentam nenhuma correlação com o peso do baço. A ocorrência de varizes de fundo gástrico nos pacientes com baço inferior a 500 gramas foi maior $(41,2 \%)$ do que os outros dois grupos, sem que houvesse significância estatística. Isto poderia ser decorrente da presença de colaterais mais calibrosas (vasos curtos do estômago), reduzindo a pressão portal.

3. O hiperesplenismo tem relação direta com o peso do baço. À medida que aumenta o peso do baço aumenta a prevalência de hiperesplenismo, chegando a 76,9\% dos pacientes com baço pesando acima de 1.000 gramas. Estes dados foram corroborados com os exames hematológicos que confirmaram uma diminuição estatisticamente significativa nos valores de hematócrito, leucócitos, linfócitos, plaquetas e tempo de protrombina, nos pacientes do Grupo 3 no pré-operatório. Ou seja, o aumento do baço determina um maior consumo dos componentes hematológicos na esquistossomose mansônica na forma hepatoesplênica.

4. O calibre da veia porta também apresenta relação com o peso do baço, aumentando progressivamente à medida que o peso do baço aumenta. A esplenectomia reduziu significativamente o calibre da veia porta, de 1,1 para $0,8 \mathrm{~cm}$ nos pacientes do Grupo 1 , e de 1,4 para 0,9 e de 1,5 para $1 \mathrm{~cm}$ nos pacientes do Grupo 2 e 3 , respectivamente. Domingues ${ }^{20}$ apresentou uma correlação entre o tamanho do baço e a pressão portal. A média da pressão portal de 21 pacientes portadores de esquistossomose hepatoesplênica com antecedentes de hemorragia digestiva foi de $37,4 \mathrm{~cm}$ de água. Nos pacientes em que o tamanho do baço excedia $10 \mathrm{~cm}$ do rebordo costal a pressão portal foi em média $42,2 \mathrm{~cm}$ de água ${ }^{20}$.

5. A recidiva do sangramento foi maior no grupo de pacientes em que o baço apresentava peso inferior a 500 
gramas. Apesar deste dado não ter respaldo estatístico é um indício de que este grupo de pacientes se beneficiaria menos da esplenectomia do que os pacientes com baço pesando mais de 500 gramas. $\mathrm{O}$ aumento da casuística é um dado importante na análise desta tendência. Domingues ${ }^{20}$ demonstrou que, mesmo em baços com dimensões pouco aumentadas, a esplenectomia determina uma redução imediata de cerca de $27 \%$ da pressão portal.

6. A incidência de trombose da veia porta também não apresentou relação com o peso do baço. A esplenectomia em baços acima de 1.000 gramas determinou um índice de trombose de $5,1 \%$, semelhante ao grupo de pacientes com baço abaixo de 500 gramas. A redução brusca do hiperfluxo determinada pelo grande volume de baço parece não interferir com a incidência de trombose portal pós-esplenectomia. Nos pacientes em que o tamanho do baço excedia $10 \mathrm{~cm}$ do rebordo costal, Domingues identificou uma redução de $31,2 \%$ nos níveis da pressão portal ${ }^{20}$. No entanto, esta redução parece não ter influenciado na incidência de trombose portal.

7. O grau de fibrose periportal no fígado também não apresentou correlação com o peso do baço. Este dado corrobora os achados de Andrade et al. ${ }^{21}$ que também não identificou relação do baço com o grau de fibrose periportal do fígado.
8. A mortalidade no período de pós-operatório imediato só ocorreu no Grupo 2. Não houve mortalidade no pósoperatório imediato no grupo de pacientes com baço acima de 1.000 gramas. O hiperesplenismo, presente de maneira estatisticamente maior neste grupo, não determinou complicações letais. Consideramos que a ligadura imediata da artéria esplênica contribui para que durante o ato cirúrgico as repercussões da pancitopenia fossem minimizadas ${ }^{22}$.

9. As alterações hematológicas evidenciadas no período pré-operatório nos pacientes do Grupo 3 foram totalmente revertidas nos exames colhidos no período pósoperatório tardio.

10. Não identificamos nenhuma alteração nos valores pré ou pós-operatório de glicose, uréia, creatinina, albumina, transaminases e nas bilirrubinas, nos grupos analisados.

Com os nossos resultados podemos concluir que o peso do baço apresenta relação direta com a ocorrência de hiperesplenismo pré-operatório, com o calibre da veia porta e com a permanência hospitalar pós-operatória. Não encontramos, entretanto, relação entre o peso do baço com a incidência de varizes de fundo gástrico, com a recidiva de sangramento digestivo, com a trombose da veia porta ou com o grau de fibrose periportal.

\begin{abstract}
Background: Searching for that risk factors that could commit surgical treatment of patient with schistosomiasis, the authors analyzed the spleen as a prognostic factor. Methods: Between 1992 and 1998, 114 patients underwent splenectomy with ligation of the left gastric vein (LLGV) and devascularisation of the great stomach curvature, followed by post-operative endoscopic sclerotherapy for the treatment of hepatic-splenic schistosomiasis with previous gastrointestinal haemorrhages. A clinical/laboratorial analysis was performed. Patients were divided into 3 groups according to the weight of the spleen, in the moment of the accomplishment of the histologycal cuts: GROUP I - spleen smaller than 500 grams (17); GROUP II - spleen between 500 and 1000 grams (58) and; GROUP III - spleen greater than 1000 grams (39). Results: Patients of GROUP I presented rebleeding rate of $17,6 \%$, thrombosis of the portal vein of 5,9\% and there was not mortality. The incidence of preoperative hipersplenism was 29,4\% and the caliber of the portal vein was 1,1 cm. In GROUP II the rebleeding rate was of $15,5 \%$, thrombosis of the portal vein of $10,3 \%$ and the mortality incidence of hiperplenism of 4 patient $(6,9 \%)$, 2 patients in the immediate postoperative period and 2 in the late followup (hepatocarcinoma and digestive hemorrhage). The hipersplenism incidence was of 53,4\% and preoperative mean caliber of the portal vein was 1,4cm. In GROUP III the rebleeding incidence was 12,8\%, thrombosis of the portal vein 5,1\% and a late mortality of 2 patient (linfoma and miocardium infarct). The hipersplenism incidence was of $76,9 \%$ and the caliber of the portal vein was of 1,5 cm. Conclusions: Weight of the spleen presents relationship with preoperative hipersplenism, with portal vein caliber and postoperative hospitalar stay. There was no relationship with fundus gastric varices, rebleeding rate, portal vein thrombosis, degree of periportal fibrosis and biochemical data.
\end{abstract}

Key Words: Hepatosplenic shistosomiasis; Splenectomy; Spleen; Risk factor; Portal vein thrombosis.

\title{
REFERÊNCIAS
}

1. Ferraz AAB, Lopes EPA, Bacelar TS et al. Tratamento cirúrgico da hipertensão portal esquistossomótica no HC-UFPE - Análise de 131 casos. Rev.Col.Bras.Cir., 2000, 27(5):332-337.
2. Ferraz AAB, Arruda SMB, Bacelar TS et al. Trombosis de la vena porta despús de esplenectomia para hipertension portal esquistosómica. Rev.Colombiana Cir. 2000, 15(3):1-7. 
3. Magalhâes Filho A, Coutinho-Abath E. Lesôes esplênicas iniciais na esquistossomose mansônica humana. Rev.Inst.Med.Hyg., 1960, 2:251.

4. Magalhâes Filho A, Coutinho-Abath E. Splenic reactions in swiss mice to single and multiple infections with Shistosoma mansoni. Am.J.Trop.Med.Hyg., 1961, 10:356-361.

5. Magalhães Filho A, Figueiredo Silva J. Patologia e patogenia da esquistossomose mansônica. In. Malta J. Esquistossomose mansônica. Recife. Editora Universitária. 1994. Pp. 61-80.

6. Kelner S, Ferreira PR, Dantas A et al. Ligadura de varizes esôfago-gástricas na hipertensão porta esquistossomótica: evolução de 25 anos. Rev.Col.Bras.Cir., 1982, 9:140-146.

7. Silveira MJ, Kelner S. Alterações hemodinâmicas do sistema porta da esquistossomose hepatoesplênica. In. Kelner S, Silvera M. Varizes do esôfago na esquistossomose mansônica. Recife. Editora Universitária. 1997. Pp. 79-86.

8. Santos OC. Estudos morfométricos das áreas linfocitárias no folículo de baços em esquistossomóticos hepatoesplênicos. Tese de Mestrado. Universidade Federal de Pernambuco. 1991.

9. Ferraz EM, Bacelar TS, Ferraz AAB et al. Infecção de ferida pós-esplenectomia em pacientes com ou sem esquistossomose hepatoesplênica. Rev. Col. Bras. Cir., 1991, 18(3):75-79.

10. Coutinho A, Domingues ALC. Specific treatment of advanced Shistosomiasis liver disease in man: Favourable results. Mem. Inst. Oswaldo Cruz, 1992, 82(Sup. IV):335-340.

11. Domingues ALC. Tratamento da esquistossomose hepatoesplênica com praziquantel: Aspectos evolutivos. Tese Mestrado. Universidade Federal de Pernambuco. 1986.

12. Cury AA. Hipertensão portal esquistossomótica: História natural. In. Abrantes W. Hipertensão portal - Estado atual. Clinica Brasileira de Cirurgia, Colégio Brasileiro de Cirurgiões, 1995, 2(1):121-136.

13. Coelho RB. Lesões hepáticas secundárias. In: Coelho RB. Anatomia patológica das afecções hepáticas. Recife, Editora Universitária da UFPE. 1971. P.59-77.

14. Eichner ER. Splenic function: normal, too much and too little. Am. J. Med., 1979, 66:311.

15. Sheldon GF, Croom RD, Meyer AA. The spleen. In: Sabiston DC. Textbook of Surgery.Philadelphia. W.B.Sauders. 14 ed. 1991. Pp. 1108-1133.

16. Ferraz EM, Ferraz AAB. Tratamento cirúrgico da hipertensão portal esquistossomótica. In: Malta J. Esquistossomose Mansônica. Recife, 1994. Ed. Universitária da UFPE. Pp 235-249.

17. Abrantes WL. Análise crítica da cirurgia de hipertensão portal esquistossomótica. In: Oliveira e Silva A, D’Albuquerque LC. Hepatologia clínica e cirúrgica. São Paulo. SARVIER, 1986. Pp. 671-682.
18. Kelner S. Avaliação da esplenectomia e ligadura intra-esosafiana das varizes do esôfago na esquistossomose mansônica. Tese para Professor Catedrático da Faculdade de Medicina da Universidade do Recife. 1965.

19. Silveira GK. Efeito da esplenectomia sobre o hormônio de crescimento em pacientes portadores de esquistossomose hepatoesplênica e hipodesenvolvimento somático. Tese de Mestrado. Universidade Federal de Pernambuco. 1976.

20. Domingues ALC. Ultra-sonografia na esquistossomose mansônica hepatoesplênica: avaliação da intensidade da fibrose periportal e da hipertensão porta. Tese Doutorado em Medicina - Universidade Federal de Pernambuco. 1998.

21. Andrade ZA, Silva LM, Souza MM et al. Role of the spleen on the pathogenesis of schistosomal periportal (pipestem) fibrosis of the liver: an experimental approach. Am. J. Trop. Med. Hyg., 1998, 59(4):557562.

22. Nelson EW, Mone MC. Splenectomy in high risk patients with splenomegaly. Am. J. Surg., 1999, 178(6):581596.

Endereço para correspondência:

Av. Beira Rio, 240, apto 2501 - Madalena

50750-400 - Recife-PE

FAX (081)271.15.26

E.mail:aabf@truenet.com.br 